18

\title{
Идентификация NV-центров в синтетических флуоресцентных наноалмазах и контроль дефектности кристаллитов методом электронного парамагнитного резонанса
}

\author{
(C) B.Ю. Осипов ${ }^{1,2}$, К.В. Богданов ${ }^{2}$, F. Treussart ${ }^{3}$, A. Rampersaud ${ }^{4}$, A.B. Баранов ${ }^{2}$ \\ ${ }^{1}$ ФТИ им. А.Ф. Иофрфре РАН, \\ 194021 Санкт-Петербург, Россия \\ 2 Университет ИТМО, \\ 197101 Санкт-Петербург, Россия \\ ${ }^{3}$ Université Paris-Saclay, CNRS, ENS Paris-Saclay, CentraleSupélec, LuMIn, \\ 91190, Gif-sur-Yvette, France \\ ${ }^{4}$ Columbus Nanoworks, Columbus, \\ 43212 Ohio, United States \\ e-mail: osipov@mail.ioffe.ru, kirw.bog@gmail.com
}

Поступила в редакцию 29.10.2021 г.

В окончательной редакции 06.11.2021 г.

Принята к публикации 07.11.2021 г.

Исследованы $100 \mathrm{~nm}$ частицы синтетического алмаза с большим (> $4 \mathrm{ppm})$ количеством азот-вакансионных $\left(\mathrm{NV}^{-}\right)$центров. Последние обнаруживают линии, связанные с запрещенными $\Delta m_{s}=2$ и разрешенными $\Delta m_{s}=1$ переходами на спектрах электронного парамагнитного резонанса (ЭПР) основного состояния $\mathrm{NV}^{-}$центра. Интенсивность люминесценции частиц в диапазоне $600-800 \mathrm{~nm}$ увеличивается с дозой облучения $5 \mathrm{MeV}$ электронами и коррелирует с интегральной интенсивностью линии ЭПР с $g$-фактором $g=4.27$. Эта величина используется для оценки концентрации $\mathrm{NV}^{(-)}$центров и отбора алмазных порошков с наибольшей интенсивностью флуоресценции. Зависимость пиковой интенсивности ЭПР сигнала $\Delta m_{s}=2$ перехода $\mathrm{NV}^{(-)}$центра от микроволновой мощности имеет вид кривой с насыщением и последующим спадом, и достаточно хорошо характеризует кристаллическое качество локального окружения исследуемых центров в этих частицах. Интенсивность $x, y \Delta m_{s}=1$ перехода (при $\sim 281.2 \mathrm{mT}, 9.444 \mathrm{GHz}$ ) оказывается более чувствительной к изменению размера частицы в субмикронном диапазоне и появлению приповерхностных дефектов, полученных в ходе механической обработки.

Ключевые слова: люминесценция, азот-вакансионные центры, синтетический алмаз, нанокристаллы, электронный парамагнитный резонанс.

DOI: $10.21883 /$ OS.2022.02.52004.2872-21

\section{Введение}

Флуоресцентные микро- и наноалмазы с азотвакансионными (NV) центрами являются новым перспективным инженерным материалом для инновационных информационных применений в телекоммуникационной сфере, нанофотонике и биомедицине [1-4]. Потенциальные применения этих материалов требуют дальнейшего развития неоптических средств диагностики центров, ответственных за люминесценцию в этих материалах.

В синтетических наноалмазах размерного диапазона до $100-130 \mathrm{~nm}$ недавно методом электронного парамагнитного резонанса (ЭПР) были обнаружены и идентифицированы сигналы, обусловленные $\mathrm{NV}^{(-)}$триплетными центрами (так называемые центры W15) [5,6]. Впервые центр W15 наблюдался около 40 лет назад в облученных электронами или протонами объемных кристаллах алмаза, содержащих примеси азота [7]. Вследствие особенностей своей микроскопической структуры $\mathrm{NV}^{(-)}$центр имеет спин $S=1$ и является оптически активным - является источником люминесцентного излучения с безфононной линией при $638 \mathrm{~nm}[8,9]$. Специфические ЭПР сигналы, соответствующие $\mathrm{NV}^{(-)}$центрам, обусловлены так называемыми запрещенными $\left(\Delta m_{s}=2\right)^{1}$ переходами между зеемановски-расщепленными уровнями энергии триплетного состояния центра $S=1$ в магнитном поле [10]. Исследование люминесценции этих центров в нано- и микрочастицах является актуальной задачей в связи с возможными применениями излучения $\mathrm{NV}^{(-)}$ центров в нанофотонике, оптических защищенных каналах связи и биомедицинских исследованиях на клеточном уровне. В данной работе исследуются $\mathrm{NV}^{(-)}$ центры в $100 \mathrm{~nm}$ флуоресцентных частицах, полученных из микрокристаллов синтетического НРНT Ib алмаза путем размола, последующего облучения пучком высокоэнергетических электронов и отжига. Результаты этих исследований, выполненных оптическими методами и с помощью метода ЭПР, сопоставляются между

\footnotetext{
1 Здесь $\Delta m_{s}$ - изменение проекции магнитного момента спина $S=1$ на направление магнитного поля, т.е. изменение магнитного квантового числа электронного состояния.
} 
собой. Поверхность и околоповерхностные слои наночастиц толщиной до 5-10 $\mathrm{nm}$ являются источниками гигантского числа дефектов (с двумерной плотностью $\sim 0.2-1 \mathrm{~nm}^{-2}$ ) как точечных, так и кооперативных, образующихся там вследствие раскола кристаллической решетки при механическом размоле и в результате воздействия на приповерхностные слои больших сдвиговых и растягивающих напряжений от размалывающих элементов $[11,12]$. Проблематика механически нарушенного слоя в алмазных частицах недавно рассматривалась в контексте сдвига электронной плотности от донорного азота к центрам внутри нарушенного слоя, приводящего к формированию непарамагнитного азота с нулевым магнитным моментом [13]. Поверхностные центры также могут менять зарядовое состояние оптических центров и парамагнитных дефектов внутри наночастиц, и „выключать“ часть из них из анализируемого явления или процесса. С уменьшением диаметра частиц роль поверхностных дефектов и дефектов, расположенных в механически нарушенном слое, возрастает. При уменьшении размера алмазных частиц, примерно начиная со 150-200 nm диапазона, свойства кристаллов начинают меняться. Так, ширина рамановской линии алмаза в наночастицах со средним размером 75-100 nm увеличена на $2.0-2.7 \mathrm{~cm}^{-1}$ по сравнению с этой величиной в объемном кристалле алмаза, из которого они были получены. В полученных размолом частицах со средним размером $25 \mathrm{~nm}$ рамановская линия алмаза уширяется еще больше на $3.9 \mathrm{~cm}^{-1}$ [14]. Такой тренд, проявляющийся в больших дополнительных уширениях $\left(\sim 4 \mathrm{~cm}^{-1}\right)$ рамановской линии алмаза при малых размерах частиц, и соответственно в незначительных уширениях линии $\left(<1 \mathrm{~cm}^{-1}\right)$ при больших размерах ( 180 nm), означает, что оптические свойства частиц, связанные с фононной подсистемой, начинают отличаться от объемных параметров уже при размерах $\leq 400 \mathrm{~nm}$. Немаловажную роль в этом играют коллективные нарушения в кристаллической решетке, организованные на большом масштабе (от 30-50 nm и выше). Среди них дислокации, мультивакансии, нанопоры, участки с большими механическими напряжениями и т.д. О влиянии механических напряжений на структуру электронных уровней $\mathrm{NV}^{(-)}$центров в алмазе и наноструктурах на его основе сообщалось в $[15,16]$. Следовательно, можно было бы ожидать, что дефекты и трещины, наведенные в приповерхностном слое кристаллов размолом, будут изменять параметры электронных уровней части близлежащих $\mathrm{NV}^{(-)}$центров за счет создаваемых ими механических напряжений в решетках алмазных частиц размером $\leq 100 \mathrm{~nm}$. Главной отличительной особенностью данной работы является то, что для анализа кристаллического качества решетки алмаза в ближайшей окрестности ${ }^{2}$ от $\mathrm{NV}^{(-)}$центров

\footnotetext{
2 Порядка длины когерентности фонона в решетке алмаза при комнатной температуре или среднего расстояния между $\mathrm{NV}^{(-)}$центрами и другими парамагнитными дефектами, в зависимости от ситуации, зависящей в том числе и от температуры.
}

применена техника снятия характеристических кривых насыщения сигнала ЭПР этих центров в зависимости от поглощаемой микроволновой мощности. Это позволяет качественно оценить влияние дефектов и парамагнитных центров, расположенных на поверхности наночастиц, на $\mathrm{NV}^{(-)}$центры, расположенные случайно-равномерно внутри наночастицы, и оценить при каких условиях поверхность начинает сильно видоизменять характеристики $\mathrm{NV}^{(-)}$центров в решетке, связанные с излучательной рекомбинацией в оптическом или микроволновом диапазонах. Ранее эта методика была успешно апробирована и применена при изучении кристаллического качества субмикронных $(<1000 \mathrm{~nm})$ алмазов, получаемых из $5 \mathrm{~nm}$ детонационных наноалмазов методом спекания в условиях высоких давлений и температур [17]. При этом для мониторинга был выбран ЭПР сигнал основного парамагнитного центра Р1, связанного с замещающими азотными примесями в решетке алмаза и имеющего спин $S=1 / 2[18]$.

\section{Образцы и методы исследований}

Частицы флуоресцентного алмаза (fluorescent nanodiamond, FND) со средним размером $100 \mathrm{~nm}$ получались из синтетического (с примесями атомарного азота) микрокристаллического НРНТ Ib алмаза с размером зерна до $150 \mu \mathrm{m}$. Последний вначале подвергался интенсивному раздроблению и размолу, а затем выделенная из помола субмикронная фракция (средний размер $104 \mathrm{~nm}$, ширина функции распределения частиц на полувысоте $\sim 76 \mathrm{~nm}$ ) облучалась в слое толщиной несколько миллиметров пучком электронов высоких энергий $(5 \mathrm{MeV})$ и отжигалась в инертной атмосфере при $800^{\circ} \mathrm{C}$. Фракция с номиналом $100 \mathrm{~nm}$ отбиралась методом центрифугирования в водной среде. Облучение электронным пучком использовалось для создания вакансий в решетке алмаза, а последующий отжиг служил для обеспечения подвижности вакансий в решетке и их захвата на изолированные азотные примеси в результате случайного блуждания. Концентрация примесного азота в исходных НРНТ Ib микрокристаллах составляла $150 \pm 10$ ppm. Плотность электронного тока в высокоэнергетическом пучке составляла $32 \mu \mathrm{A} / \mathrm{cm}^{2}$. В процессе облучения порошкообразный материал охлаждался с помощью специального устройства для предотвращения паразитного перегрева. Материал, облученный электронами в течение интегральных периодов в 16, 32 и $40 \mathrm{~h}$, обозначен как FND-1, FND-2, FND-3. Указанные экспозиции набирались из одной, двух или трех последовательных сессий облучения длительностью 16 и $8 \mathrm{~h}$. Длительные экспозиции при облучении $100 \mathrm{~nm}$ частиц выбирались для увеличения количества наведенных вакансий и, следовательно, $\mathrm{NV}^{(-)}$центров в получаемом на выходе материале. Субмикронные $(\sim 100 \mathrm{~nm})$ частицы в прин- 
ципе не имеют существенных недостатков ${ }^{3}$ для процесса создания вакансий и NV центров в алмазе по сравнению с кристаллами размером от микрометра и выше. Это проистекает в результате того, что вероятность образования вакансией одного NV центра в отдельной монокристаллической частице со средним уровнем легирования азотом асимптотически стремится к 1 с увеличением размера кристаллита $\left(L_{c}\right)$ при $L_{c}>40-50 \mathrm{~nm}$ и зависимость от $L_{c}$ слабая в этом диапазоне [19]. Минимальная номинальная доза облучения, соответствующая наименьшей экспозиции, составляла $7 \cdot 10^{18} \mathrm{~cm}^{-2}$. С учетом разных условий охлаждения порошков в первой и последующих сессиях облучения эквивалентные дозы облучения приводили, по всей видимости, к разным количествам наводимых в материале оптических центров из-за перегрева. Образцы, прошедшие этапы облучения и отжига, далее подвергались интенсивной химической очистке в кипящих кислотах с целью удаления паразитных металлических примесей, главным образом железосодержащих комплексов. Образцы изготовлялись и технологически обрабатывались компанией Columbus Nanoworks (Columbus, Ohio, США).

Спектры ЭПР образцов регистрировались при комнатной температуре с помощью ЭПР спектрометра JEOL JES-FA 300 (Япония) на частоте $9.5 \mathrm{GHz}$. Спектры сигналов ЭПР с $g$-факторами в диапазоне 4.00-4.30 записывались при малой мощности микроволнового излучения $0.003 \mathrm{~mW}$, амплитуде модуляции $0.2 \mathrm{mT}$, частоте модулирующего магнитного поля $100 \mathrm{kHz}$, числе накоплений сигнала 10-16. Постоянная времени составляла $0.03 \mathrm{~s}$, полное время записи спектра для одного прохода в интервале развертки магнитного поля от 130 до $200 \mathrm{mT}-120 \mathrm{~s}$. При малых микроволновых мощностях $\left(P_{\mathrm{MW}}<0.01 \mathrm{~mW}\right)$ искажений формы ЭПР сигналов от насыщения зарегистрировано не было и рост сигнала с увеличением $P_{\mathrm{MW}}$ был практически пропорционален $\sqrt{P_{\mathrm{MW}}}$. Концентрации центров $\mathrm{NV}^{(-)}$ оценивались путем двойного интегрирования сигнала ЭПР $(g=4.27)$, снятого при малой микроволновой мощности $\left(P_{\mathrm{MW}} \approx 0.003 \mathrm{~mW}\right)$, и отнесения полученной величины к весу порошкообразного образца. Для получения абсолютной величины концентрации $\mathrm{NV}^{(-)}$центров в единицах „ррт“ в качестве эталона для сравнения использовались стандарты с известной концентрацией парамагнитных центров $S=1$ и $S=1 / 2$ [10,20] и методика, описанная в [10]. При микроволновых мощностях свыше $0.03 \mathrm{~mW}$ отмечалось отклонение от линейного хода зависимости пиковой интенсивности $\left(I_{p p}\right)^{4}$ сигнала ЭПР $\left(g_{1}=4.27\right)$ от корня из микроволновой мощности $\sqrt{P_{\mathrm{MW}}}$. Для построения кривых насыщения

\footnotetext{
${ }^{3}$ К недостаткам субмикронного алмазного порошка следует отнести худшую теплопроводность и повышенную летучесть по сравнению с порошками кристаллитов микронных размеров.

4 Пиковая интенсивность ЭПР сигнала $\left(I_{p p}\right)$ вычисляется в соответствии с определением этой величины, как половина расстояния (по вертикали) между ординатами максимума и минимума на спектре первой производной ЭПР сигнала. Здесь мы пренебрегаем сильной асимметрией линии ЭПР $\left(g_{1}=4.27\right)$.
}

пиковой интенсивности сигнала ЭПР $I_{p p}\left(g_{1}=4.27\right)$ от $\left(\sqrt{P_{\mathrm{MW}}}\right)$ дополнительно регистрировались спектры ЭПР для целого ряда значений $P_{\mathrm{MW}}$ в диапазоне от 0 до $200 \mathrm{~mW}$ для образцов FND-1, FND-2 и микронного размера кристаллов НРНТ Ib алмаза с $\mathrm{NV}^{(-)}$центрами. В целях лучшего сопоставления и сравнения трендов $I_{p p}$ $\left(\sqrt{P_{\mathrm{MW}}}\right)$ при больших значениях $P_{\mathrm{MW}}$ кривые насыщения для разных образцов нормировались на величину $I_{p p}$ $\left(P_{\mathrm{MW}}=0.003 \mathrm{~mW}\right): I_{p p}\left(P_{\mathrm{MW}}\right) / I_{p p}\left(P_{\mathrm{MW}}=0.003 \mathrm{~mW}\right)$.

Для одновременного сопоставления и анализа ЭПР сигналов от запрещенных $\left(\Delta m_{s}=2\right)$ и разрешенных $\left(x, y \Delta m_{s}=1\right)$ переходов в $\mathrm{NV}^{(-)}$центрах разных образцов дополнительно регистрировались спектры ЭПР в широком диапазоне магнитных полей от 120 до $320 \mathrm{mT}$ и строилась зависимость отношения пиковых интенсивностей обоих сигналов $I_{p p}^{\text {all }} / I_{p p}^{\text {forb }}$ от $\sqrt{P_{\mathrm{MW}}}$. Указанные спектры снимались при тех же параметрах съемки, отмеченных выше.

Спектры люминесценции измерены с помощью микрорамановской установки inVia (Renishaw, Великобритания) с использованием возбуждающего лазерного излучения на длине волны $532 \mathrm{~nm}$ и $50^{\times}$микрообъектива, фокусирующего излучение в пятно диаметром $\sim 2 \mu \mathrm{m}$ на поверхности образца. Люминесцентное излучение собиралось в геометрии обратного рассеяния с поверхности образца. Флуоресцентные изображения частиц, осажденных из капли водной суспензии, были получены с помощью эпифлуоресцентного инвертированного микроскопа Nikon Eclipse TiS с использованием оптического фильтра Texas Red Cube (возбуждение $540-580 \mathrm{~nm}$, проходящее излучение 600-660 nm). Изображения получены при $10 \mathrm{~ms}$ экспозиции с помощью $10^{\times}$микрообъектива. Оптические и флуоресцентные изображения изолированных частиц FND также были получены с помощью многофункционального видео-микроскопа собственной конструкции (в боксеинкубаторе от компании OKOLab, Италия), работающего в двух режимах: дифференциального интерференционного контраста и флуоресцентной микроскопии полного внутреннего отражения (TIRF). В первом варианте использовался проходящий белый свет галогеновый лампы (OSRAM HLX 64623), а контраст изображения подбирался взаимным вращением поляризатора и анализатора в рабочей схеме. Во втором варианте использовалось $561 \mathrm{~nm}$ возбуждающее излучение от твердотельного лазера непрерывного действия (SLIM561-100, Oxxius S.A., Lannion, Франция), дихроический расщепитель пучка с постоянным 97\% пропусканием выше края при $561 \mathrm{~nm}$ (zt561rdc, Chroma Corporation) и полосовой фильтр (FF01-697/75, Semrock), пропускающий излучение в полосе $\sim 75 \mathrm{~nm}$ в красной области спектра. Мощность лазерного пучка, измеренная перед входом в оптическую систему микроскопа, составляла 8-10 mW. В обоих случаях использовался иммерсионный $100^{\times}$микрообъектив (CFI Apo TIRF $100^{\times}$, $\mathrm{NA}=1.49$, Oil, Nikon, Япония). Частицы осаждались путем нанесения микрокапли алмазной суспензии на 
$a$

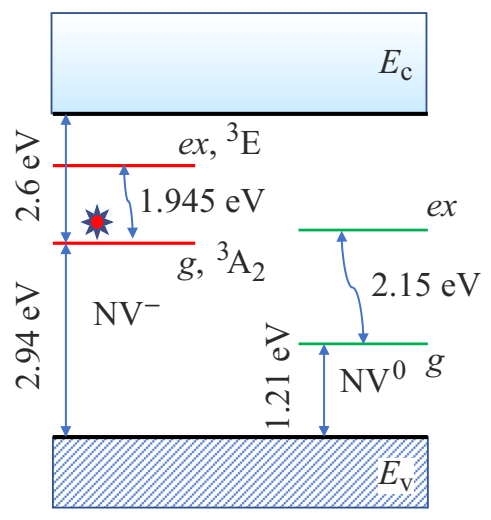

$b$

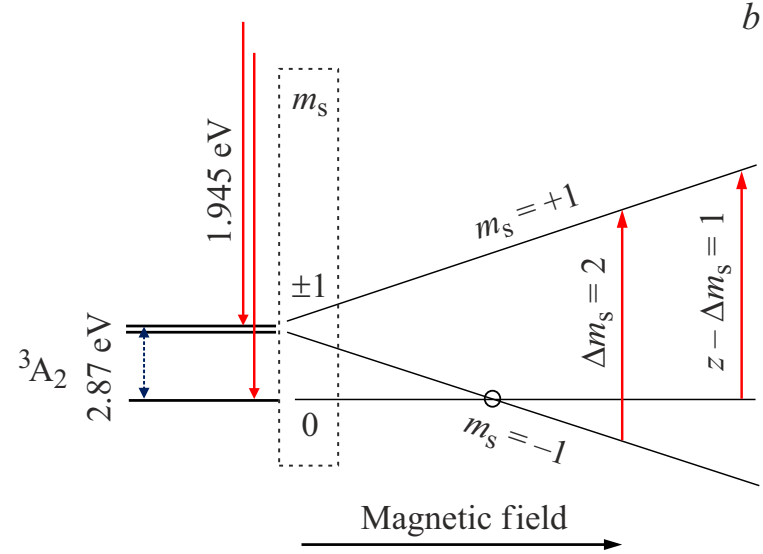

Рис. 1. Схема основных энергетических уровней центров $\mathrm{NV}^{(-)}$и $\mathrm{NV}$ в запрещенной зоне алмаза $(a)$ и зеемановское расщепление уровней основного состояния ${ }^{3} \mathrm{~A}_{2} \mathrm{NV}^{(-)}$центра в магнитном поле $(b)$. Обозначения: $g$ - основное состояние, ех - возбужденное состояние, $E_{c}-$ край зоны проводимости, $E_{v}-$ край валентной зоны, $m_{s}-$ магнитное квантовое число уровня энергии в триплетном центре.

вращающуюся подложку толщиной $\sim 170 \mu \mathrm{m} \quad(80 \mathrm{~s}$, $2000 \mathrm{rpm} / \mathrm{s}$, устройство SPIN150, АРT GmbH, Германия), предварительно обработанную в кислородной плазме в устройстве Femto $40 \mathrm{KHz}$ (Diener/Femto Plasma Cleaner, Германия) в течении $10 \mathrm{~min}$ при давлении $0.05 \mathrm{mbar}$. EMCCD камера (iXon-DU885, Andor Technology), используемая для регистрации изображений, охлаждалась до $-79.8^{\circ} \mathrm{C}$. Размеры регистрируемых изображений составляли $\sim 80 \times 80 \mu \mathrm{m}(1004 \times 1002$ pixels $)$. Размер одного пикселя в плоскости образца составлял $\sim 80 \mathrm{~nm}$.

\section{Экспериментальные результаты и обсуждение}

\section{Электронный парамагнитный резонанс}

$\mathrm{NV}^{(-)}$центры, расположенные в объеме алмазных частиц, и мультивакансии, обычно появляющиеся на поверхности частиц, подверженных размолу и/или другим механическим повреждениям, являются триплетными центрами со спином $S=1$. Спин-гамильтониан $\mathrm{NV}^{(-)}$центра в магнитном поле $\mathbf{B}\left(B_{x}, B_{y}, B_{z}\right)$ имеет следующий вид [21,22]:

$$
H=\hat{g} \mu_{\mathrm{B}} \mathbf{B} \cdot \mathbf{S}+D\left[S_{z}^{2}-\frac{1}{3} S(S+1)\right]+E\left(S_{x}^{2}-S_{y}^{2}\right),
$$

Здесь $\mathbf{S}$ - спиновый оператор, $S_{z}$ - оператор, связанный с $z$-компонентой в $\mathbf{S}$, задаваемой осью симметрии NV центра, т.е. направлением $\langle 111\rangle$ в решетке кристалла, $S_{x}, S_{y}$ - операторы проекций спина-1 на координатные оси $x$ и $y, \hat{g}-g$-тензор (или $g$-фактор спина электрона $\left.g_{x x}=g_{y y}=g_{z z}=2.0028 \pm 0.0003\right), D-$ параметр тонкой структуры, связанный с расщеплением уровня в нулевом магнитном поле (синглет-триплетная щель), $E$ - параметр, связанный с расщеплением, индуцированным напряжениями в кристаллической решетке,
$\mu_{\mathrm{B}}$ - магнетон Бора. По факту параметр $D$ также зависит от напряжений, в том числе и термическииндуцированных напряжений в решетке [1,15,21]. Запись спин-гамильтониана в формуле (1) приближенная и не учитывает сверхтонкие и квадрупольные взаимодействия, в частности взаимодействия электронных спинов $\mathrm{NV}^{(-)}$центров с магнитными моментами ядер ${ }^{14} \mathrm{~N}$ $\left(\right.$ и $\left.{ }^{13} \mathrm{C}\right)$, зеемановского члена для магнитного момента ядра атома азота ${ }^{14} \mathrm{~N}(I=1)$, и члена, связанного с квадрупольным ядерным взаимодействием. $\mathrm{NV}^{(-)}$центры являются триплетными как в основном невозбужденном $\left({ }^{3} \mathrm{~A}_{2}\right)$, так и в оптически возбужденном $\left({ }^{3} \mathrm{E}\right)$ состояниях. Обе системы уровней находятся внутри запрещенной зоны алмаза (рис. 1) [23,24]. Далее, в контексте ЭПР исследований мы будем рассматривать только основные оптически невозбужденные состояния триплетных центров (рис. $1, b)$. Расположение линий, соответствующих запрещенным $\left(\Delta m_{s}=2\right)$ и разрешенным $\left(\Delta m_{s}=1\right)$ переходам в них в микроволновом диапазоне, задается на шкале магнитного поля уникальными параметрами спингамильтонианов этих центров. Последние учитывают обменное взаимодействие между индивидуальными спинами в центрах, а параметр $D$ спин-гамильтониана, задающий ширину щели между уровнями $\left|{ }^{3} \mathrm{~A}_{2}, 0\right\rangle$ и $\left|{ }^{3} \mathrm{~A}_{2}, \pm 1\right\rangle$ центра в нулевом магнитном поле, обычно обратно пропорционален третьей степени расстояния между индивидуальными спинами $1 / 2$ в составном триплетном центре [7,25]. Здесь записи 0$\rangle$ и \pm 1$\rangle$ означают соответственно состояния с антипараллельными $\left(m_{s}=0\right)$ и параллельными $\left(m_{s}= \pm 1\right)$ ориентациями индивидуальных спинов 1/2 в триплетном центре. Индекс 3 означает триплетную структуру. Параметр $D$ спин-гамильтониана $\mathrm{NV}^{(-)}$центра был определен в конце 1970-х годов из анализа положения линий на ЭПР спектре центра [7], и затем неоднократно уточнялся [26]. Он равен $D=954 \cdot 10^{-4} \mathrm{~cm}^{-1}$ с точностью $0.3 \%$ [7]. Такое 

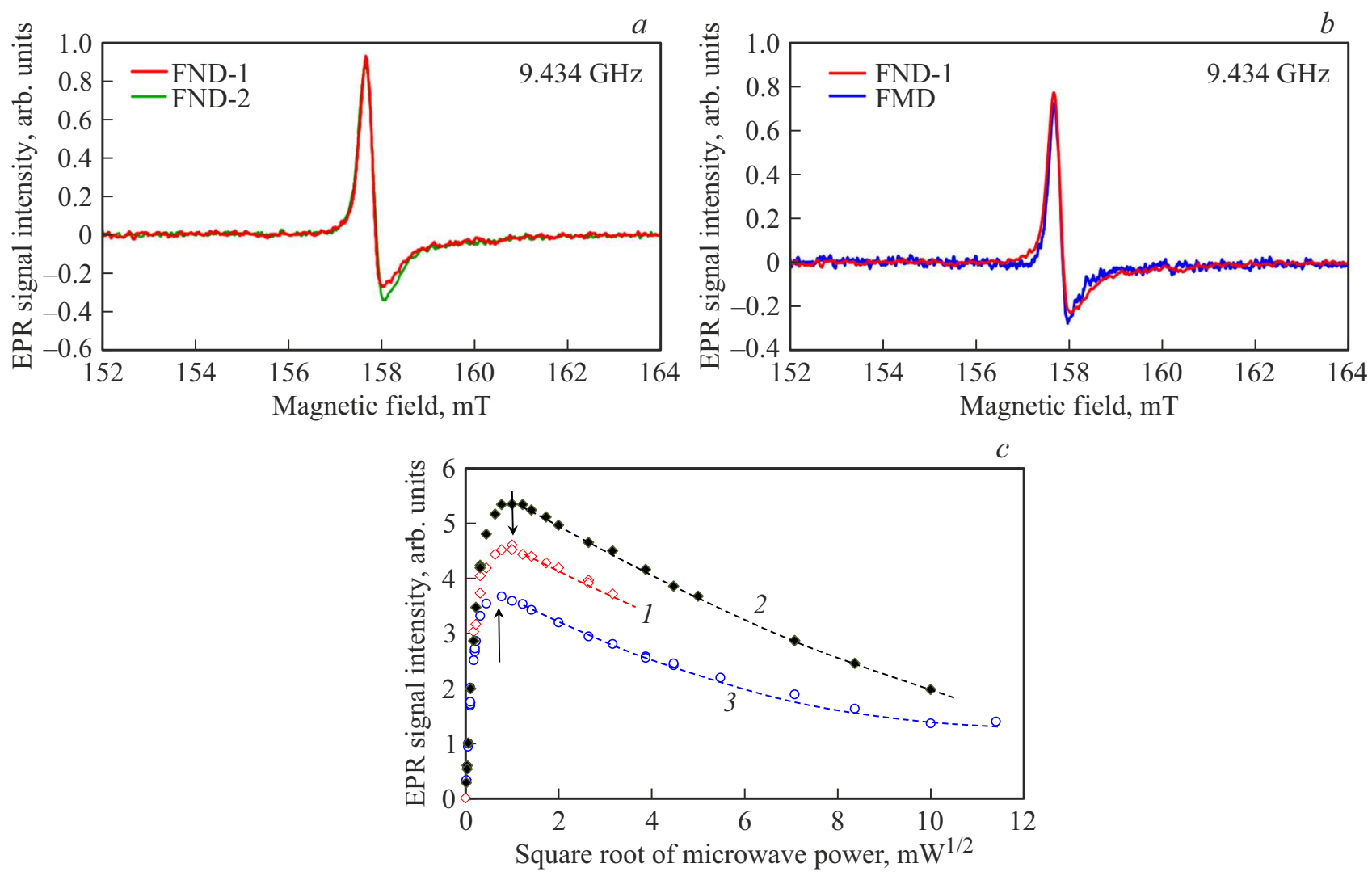

Рис. 2. Спектры ЭПР флуоресцентного синтетического НРHT Ib алмаза (FND-1, FND-2 и FMD) в парах сравнения FND-1/FND$2(a)$ и FND-1/FMD $(b)$ и кривые насыщения пиковой интенсивности сигнала ЭПР $\left(g_{1}=4.27\right)$ от корня из микроволновой мощности в диапазоне от 0 до $140 \mathrm{~mW}(c)$ для образцов FND-1 (1), FND-2 (2) и референтных микрокристаллов НРНT Ib алмаза $\mathrm{c} \mathrm{NV}^{(-)}$центрами $(3)$. Микроволновая частота $\approx 9.43 \mathrm{GHz}$. С целью лучшего сопоставления все кривые насыщения нормированы для микроволновой мощности $P_{\mathrm{MW}}=0.003 \mathrm{~mW}$.

значение примерно соответствует расстоянию $0.3 \mathrm{~nm}$ между индивидуальными спинами (неспаренными орбиталями) $S=1 / 2$ в триплетном центре. Ширина соответствующей щели равна $2.87 \mathrm{GHz}$ (или $12 \mu \mathrm{eV}$ ). Отношение $E / D$ в ряде практических случаев составляет порядка 0.002-0.003 или даже существенно меньше. Температурная зависимость $D(T)$ - слабая линейная с коэффициентом наклона около $-75 \mathrm{kHz} / \mathrm{K}$ в окрестности комнатной температуры.

Уровни $\left|{ }^{3} \mathrm{~A}_{2}, 0\right\rangle$ и $\left|{ }^{3} \mathrm{~A}_{2}, \pm 1\right\rangle$ являются основными состояниями и для излучательных оптических переходов $(h v=1.945 \mathrm{eV})$, идущих с уровней оптическивозбужденных состояний $\left|{ }^{3} \mathrm{E}, 0\right\rangle$ и $\left|{ }^{3} \mathrm{E}, \pm 1\right\rangle$, имеющих также триплетную структуру.

Спектры ЭПР образцов FND-1 и FND-2, отличающихся дозой облучения высокоэнергетическими электронами, показаны на рис. $2, a, b$ в диапазоне магнитных полей от 152 до $164 \mathrm{mT}$. В половинном ${ }^{5}$ магнитном поле при микроволновых мощностях до $1 \mathrm{~mW}$ в спек-

\footnotetext{
${ }^{5}$ Имеется в виду диапазон магнитных полей, где расположены слабые ЭПР сигналы с $g$-фактором $g \approx 4$. Специфика исследуемых образцов такова, что паразитные сигналы от железосодержащих комплексов (с $g$-фактором $g=4.25-4.27$ ), также располагающиеся в этом диапазоне, отсутствуют после химической очистки.
}

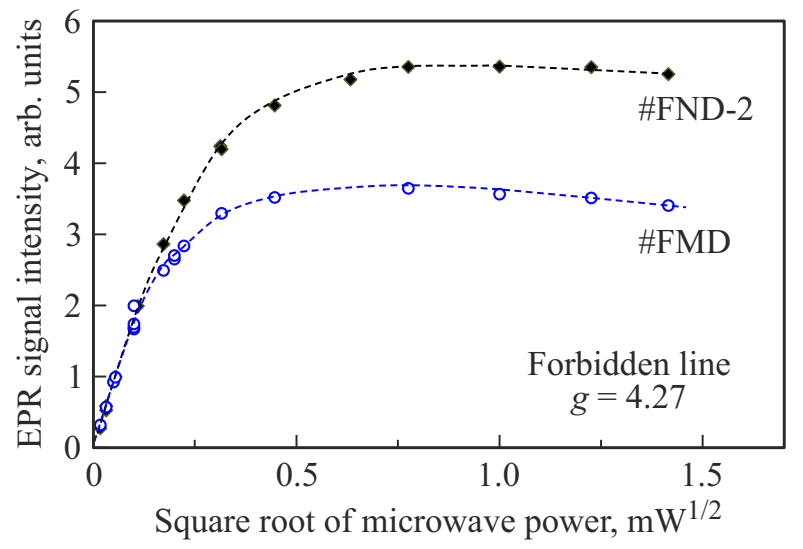

Рис. 3. Кривые насыщения пиковой интенсивности сигнала ЭПР $\left(g_{1}=4.27\right)$ от корня из микроволновой мощности в диапазоне от 0 до $2 \mathrm{~mW}$ для образцов FND-2 (черные ромбы) и FMD (синие кружки). Кривые нормированы при мощности $P_{\mathrm{MW}}=0.003 \mathrm{~mW}$.

трах присутствует только одна характерная узкая линия $g_{1}=4.27$ от $\mathrm{NV}^{(-)}$центров, интенсивность которой растет с дозой облучения, а от других триплетных 


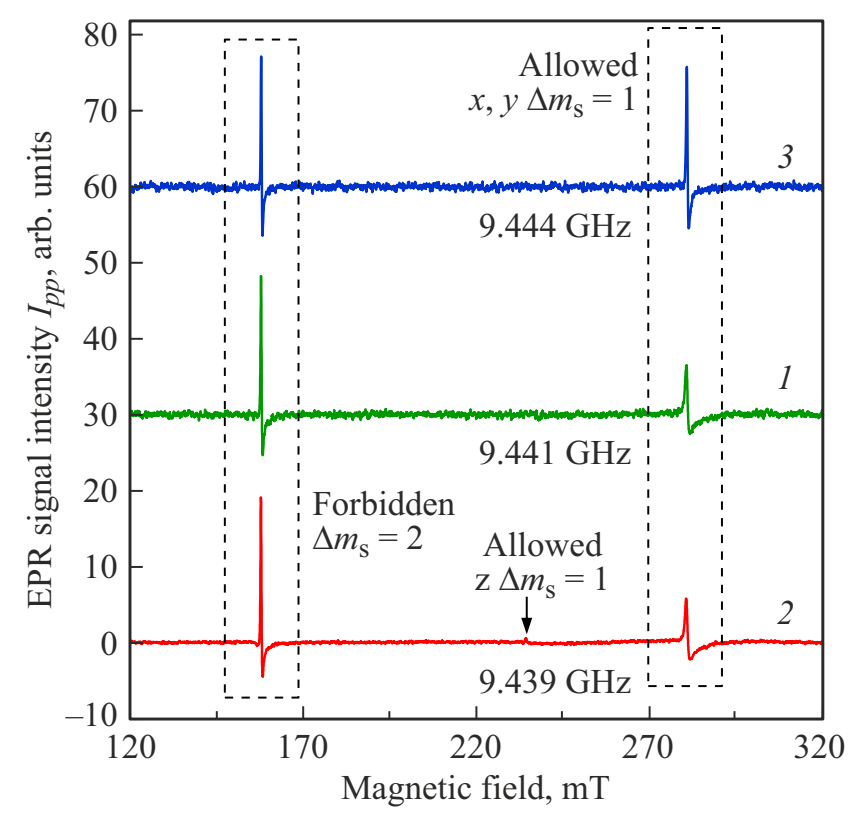

Рис. 4. Спектры ЭПР FND-1 (1), FND-2 (2) и FMD (3) в широком диапазоне магнитных полей (от 120 до $320 \mathrm{mT}$ ), демонстрирующие запрещенный $\left(\Delta m_{s}=2\right)$ и низкополевой разрешенный $\left(x, y \Delta m_{s}=1\right)$ микроволновые переходы между триплетными состояниями $\left|{ }^{3} \mathrm{~A}_{2},-1\right\rangle,\left|{ }^{3} \mathrm{~A}_{2},+1\right\rangle$ и $\left|{ }^{3} \mathrm{~A}_{2}, 0\right\rangle$ и $\left.\left.\right|^{3} \mathrm{~A}_{2},+1\right\rangle$. В целях лучшего сопоставления все спектры нормированы на пиковую амплитуду ЭПР сигнала с $g$-фактором $g=4.27$. Микроволновая частота $\approx 9.44 \mathrm{GHz}$. Верхняя граница диапазона лежит на $16 \mathrm{mT}$ ниже положения интенсивного сигнала ЭПР от дефектов парамагнитного азота (P1) в алмазе с характерной сверхтонкой структурой.

центров, например, мультивакансий, сигналы в даже более расширенном на $20 \mathrm{mT}$ диапазоне практически отсутствуют (рис. $2, a, b){ }^{6}$ Последнее означает, что размол микрокристаллов алмаза до $100 \mathrm{~nm}$ уровня еще не приводит в количественном аспекте к появлению значимого количества поверхностных дефектов (мультивакансий и оборванных связей), появляющихся при механических обработках микрокристаллов и могущих являться центрами тушения люминесценции от $\mathrm{NV}^{(-)}$ центров внутри $100 \mathrm{~nm}$ частиц за счет механизма миграции оптического возбуждения от центра к дефектной поверхности. ЭПР сигнал от $\mathrm{NV}^{(-)}$центров в образцах порошков наночастиц имеет асимметричную, нелоренцевскую форму линии, что обусловлено типом симметрии центра в кристалле, особенностями параметров $D$ и $E$ спин-гамильтониана центра, и в некоторой степени их зависимостью от локальных механических напряжений в кристаллической решетке. Отметим, что в наночастицах

\footnotetext{
${ }^{6}$ Мультивакансии, линейные кластеры небольшой протяженности, состоящие из вакансий, обусловливают наличие ЭПР сигнала с $g$ фактором $g_{2}=4.00$ в области, отстоящей по шкале магнитного поля на $\sim 10 \mathrm{mT}$ от сигнала ЭПР, соответствующего $\mathrm{NV}^{(-)}$центрам. Как и последние они являются триплетными центрами. Они, к примеру, присутствуют в поликристаллических наноалмазах динамического синтеза со средним размером в интервале $25-1000 \mathrm{~nm}$.
}

НРНТ Ib алмаза, не подвергшихся облучению электронами, сигнал ЭПР с $g$-фактором $g_{1}=4.27$ (от $\mathrm{NV}^{(-)}$ центров) отсутствует. При увеличении микроволновой мощности $P_{\mathrm{MW}}$ свыше $0.4 \mathrm{~mW}$ пиковая интенсивность сигнала $g_{1}=4.27$ для обоих образцов FND-1 и FND2 испытывает насыщение, а при мощностях свыше 2-3 mW обнаруживает уже монотонный плавный спад, простирающийся вплоть до значений $P_{\mathrm{MW}} \sim 100 \mathrm{~mW}$ (рис. 2, $c$, кривые 1 и 2). При этом ширина $\Delta H_{p p}$ линии $g_{1}=4.27$ незначительно (в пределах 20\%) увеличивается от 0.49 до $0.59 \mathrm{mT}$ в интервале $P_{\mathrm{MW}}$ от 0.001 до $100 \mathrm{~mW}$. Это указывает на реализуемую при этих значениях $P_{\mathrm{MW}}$ (за счет запрещенных $\Delta m_{s}=2$ переходов) превалирующую заселенность верхнего зеемановскиотщепленного уровня энергии $\left|{ }^{3} \mathrm{~A}_{2},+1\right\rangle$, относительно большие времена спиновой релаксации (как спинспиновой, так и спин-решеточной) и, соответственно, на высокое качество кристаллической решетки алмаза в ближайшей окрестности от $\mathrm{NV}^{(-)}$центров. ${ }^{7}$ В работе не делалось попыток определения точных времен спинспиновой и спин-решеточной релаксации для $\mathrm{NV}^{(-)}$ центров, не столько в связи с трудностями правильной интерпретации данных, сколько в связи с тем, что вид кривой насыщения $I_{p p}\left(P_{\mathrm{MW}}\right)$ качественно достаточно хорошо характеризует кристаллическое качество локального окружсения исследуемых центров. На рис. 2, с дополнительно показана кривая насыщения пиковой интенсивности сигнала ЭПР $g_{1}=4.27$ для флуоресцентных микрокристаллов алмаза FMD (с $\mathrm{NV}^{(-)}$центрами) размером в несколько микрон (кривая 3). Последняя выходит на насыщение при несколько меньших $(\sim 0.2-0.3 \mathrm{~mW})$ мощностях микроволн, чем для частиц флуоресцентного алмаза размером $100 \mathrm{~nm}$, что указывает на несколько более высокое, но не отличающееся кардинально кристаллическое качество облученных высокоэнергетическими электронами микрокристаллов НРНТ Ib алмаза, чем $100 \mathrm{~nm}$ частиц, полученных размолом из таких же кристаллов микронного размера и последующим облучением. Более ранняя тенденция к насыщению сигнала ЭПР $g_{1}=4.27$ в образце FMD (по сравнению c FND-1, FND-2) обнаруживается уже в интервале микроволновых мощностей $0.03-0.2 \mathrm{~mW}$, когда $100 \mathrm{~nm}$ и микронные образцы с $\mathrm{NV}^{(-)}$центрами демонстрируют разные отклонения (сильные и слабые) от линейной зависимости $I_{p p} \sim \sqrt{P_{\mathrm{MW}}}$ при малых мощностях (рис. 3 ). Таким образом, парамагнитные и другие решеточные дефекты, расположенные на поверхности $100 \mathrm{~nm}$ частиц, еще не оказывают сильного влияния на времена спинспиновой и спин-решеточной релаксации возбужденного микроволнами состояния $\left.\left.\right|^{3} \mathrm{~A}_{2},+1\right\rangle \mathrm{NV}^{(-)}$центров. Данная ЭПР методика может использоваться для оценки

\footnotetext{
${ }^{7}$ Например, в детонационном алмазе с содержанием $\mathrm{NV}^{(-)}$центров около $\sim 1.2 \mathrm{ppm}$ ЭПР сигнал $g_{1}=4.27$ не насыщается вплоть до микроволновой мощности в $200 \mathrm{~mW}$ в связи с большой концентрацией парамагнитных центров $S=1 / 2(1300 \mathrm{ppm})$ и других немагнитных дефектов в кристаллической решетке, в том числе связанных с присутствием азотных пар (А-центров) в количестве до 2 wt.\%.
} 
потенциальной пригодности $\mathrm{NV}(-)$ центров в качестве центров люминесценции в конкретных субмикронных кристаллах, поскольку высокое кристаллическое качество решетки алмаза вокруг центра способствует появлению длительных времен как спиновой, так и оптической ${ }^{8}$ релаксации, и наоборот. Дополнительно на рис. 4 показаны спектры ЭПР исследуемых образцов FND-1, FND-2 и референтного микрокристаллического образца FMD в широком диапазоне магнитных полей (от 120 до $320 \mathrm{mT}$ ). На всех спектрах вблизи верхней границы этого интервала присутствует сигнал, соответствующий так называемому разрешенному переходу $x, y \quad \Delta m_{s}=1$ между уровнем энергии не чувствительного к магнитному полю состояния $\left|{ }^{3} \mathrm{~A}_{2}, 0\right\rangle \mathrm{c} m_{s}=0$ и одним из уровней триплетного состояния $\mathrm{NV}^{(-)}$центра с $m_{s}= \pm 1$, претерпевающего зеемановский сдвиг в сторону больших энергий. Данный сигнал, соответствующий низкополевому $x, y$ разрешенному переходу в микроволновом диапазоне, расположен примерно при $\sim 281 \mathrm{mT}$ (при $v=9.44 \mathrm{GHz})$. Спектры, приведенные на рис. 4 , измерены при $P_{\mathrm{MW}}=1 \mu \mathrm{W}$, т. е. малой мощности. С увеличением микроволновой мощности интенсивность ЭПР линии $x, y \Delta m_{s}=1$ (для образцов FND-1, FND-2) достигает максимума, насыщается и далее падает по интенсивности еще быстрее, чем интенсивность линии ЭПР для

\footnotetext{
8 Здесь подразумевается случай оптической накачки и релаксации оптически возбужденного состояния $\mathrm{NV}^{(-)}$центра.
}

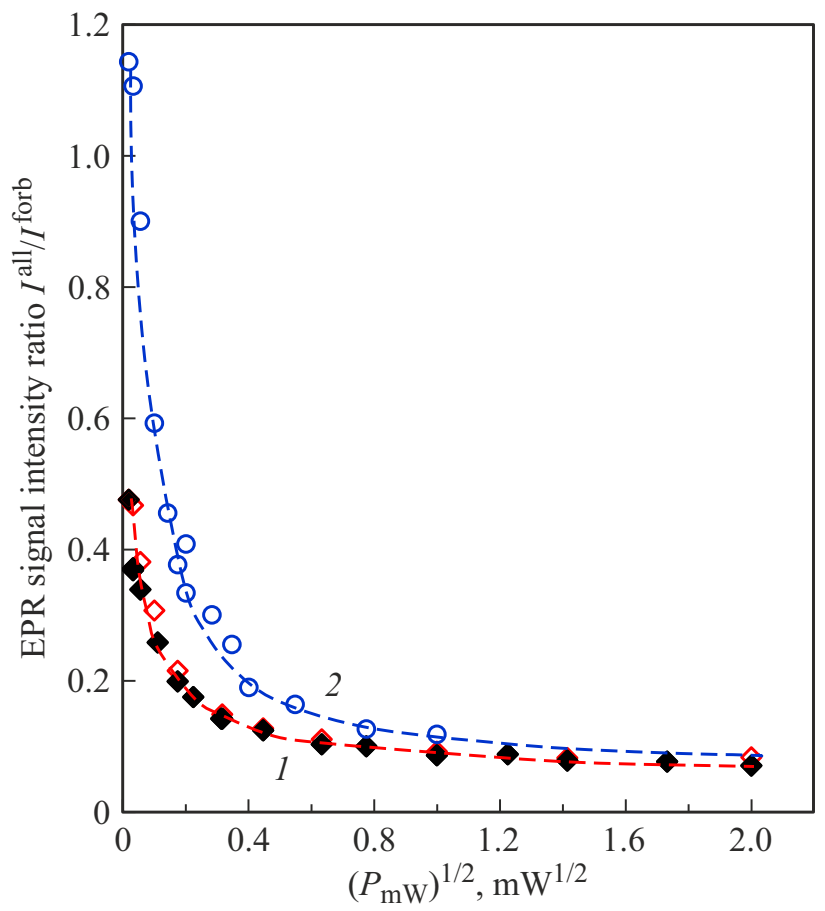

Pис. 5. Отношение пиковых интенсивностей линий ЭПР для разрешенного $\left(x, y \Delta m_{s}=1\right)$ и запрещенного $\left(\Delta m_{s}=2\right)$ переходов $\left(I_{\mathrm{all}} / I_{\text {forb }}\right)$ в зависимости от $P_{\mathrm{Mw}}$ для образцов FND-1 и FND-2 (черные и красные незаполненные ромбы) и референтных FMD микрокристаллов HPHT Ib алмаза $\mathrm{c} \mathrm{NV}(-)$ центрами (синие незаполненные кружки).

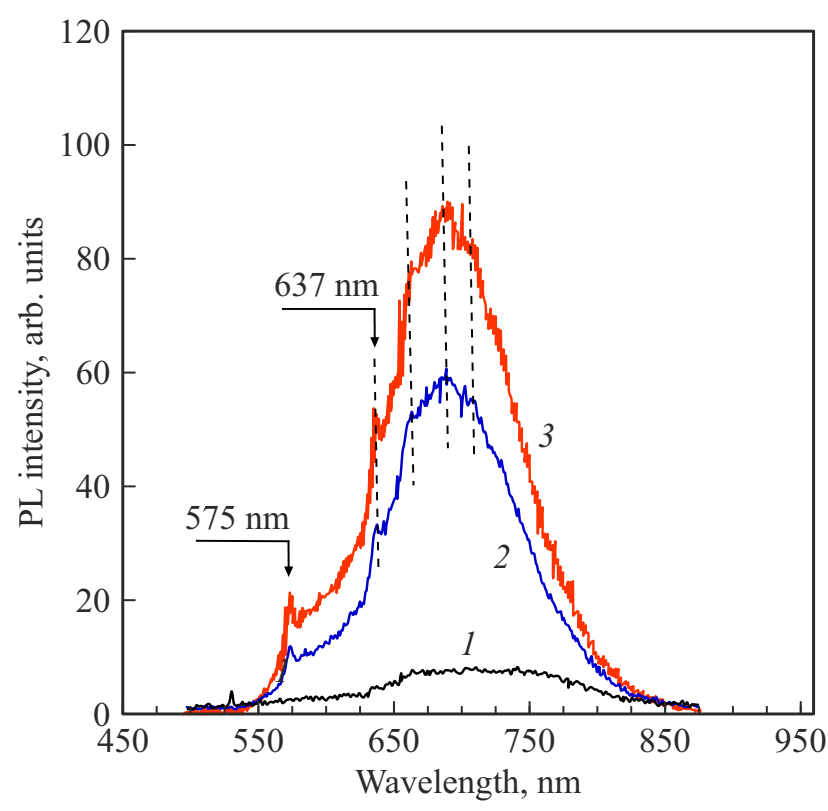

Рис. 6. Спектры люминесценции $100 \mathrm{~nm}$ частиц синтетического НРНT $\mathrm{Ib}$ алмаза $\mathrm{c} \mathrm{NV}\left(\mathrm{N}^{(-)}\right.$центрами для материалов с разной дозой облучения высокоэнергетическим электронным пучком (FND-1, FND-2, FND-3). Спектры возбуждались лазерным излучением $532 \mathrm{~nm}$.

запрещенного перехода $\Delta m_{s}=2$. При микроволновых мощностях свыше $7 \mathrm{~mW}$ первая становится практически ненаблюдаемой 9 в наночастицах размером $100 \mathrm{~nm}$ (из-за уширения и уменьшения по пиковой амплитуде), тогда как вторая (линия запрещенного перехода) наблюдается вплоть до мощностей порядка $160 \mathrm{~mW}$. На рис. 5 показано отношение $I_{\text {all }} / I_{\text {forb }}$ пиковых амплитуд линий ЭПР для разрешенного $\left(x, y \Delta m_{s}=1\right)$ и запрещенного $\left(\Delta m_{s}=2\right)$ переходов в зависимости от $P_{\mathrm{MW}}$ для образцов FND-1, FND-2 и FMD. Видно, что экспериментальные точки для FND-1 и FND-2 (черные и красные ромбы) практически совпадают и лежат на одной кривой 1 . При этом с ростом $P_{\text {MW }}$ отношение $I_{\text {all }} / I_{\text {forb }}$ быстро (в интервале $P_{\mathrm{MW}}=0-1 \mathrm{~mW}$ ) падает в обоих случаях. Вместе с тем, отношение $I_{\text {all }} / I_{\text {forb }}$ для FMD микрокристаллов HPHT Ib алмаза с $\mathrm{NV}^{(-)}$центрами характеризуется большими значениями в интервале $P_{\mathrm{MW}}=0-1 \mathrm{~mW}$ (синие кружки, рис. 5), что соответствует лучшей наблюдаемости сигнала от разрешенного $x, y \Delta m_{s}=1$ перехода в FMD в этом диапазоне мощностей. Отношение $I_{\text {all }} / I_{\text {forb }}$ при этом спадает еще быстрее (от 1.14 до 0.07 ), чем для образцов FND-1 и FND-2, в интервале мощностей $0-4 \mathrm{~mW}$ (кривая 2 на рис. 5). Это дополнительно указывает и на особую чувствительность ЭПР линии разрешенного перехода $\left(x, y \quad \Delta m_{s}=1\right)$ в $\mathrm{NV}^{(-)}$центре к дефектам и несовершенствам кристаллической решетки на поверхности наночастиц. Действие последних сводится к

\footnotetext{
${ }^{9}$ В кристаллах микронных размеров ЭПР сигнал от разрешенных переходов $\Delta m_{s}=1$ в $\mathrm{NV}^{(-)}$центрах наблюдается вплоть до мощностей микроволн порядка $100 \mathrm{~mW}$.
} 

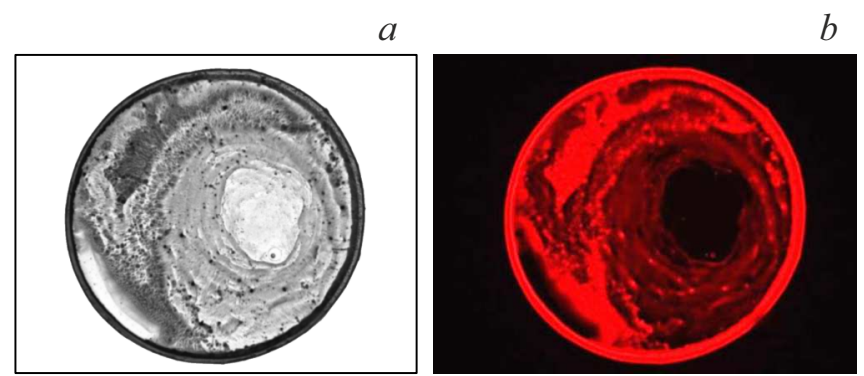

Рис. 7. Оптическое изображение высушенной капли водной суспензии наночастиц синтетического алмаза с $\mathrm{NV}^{(-)}$центрами FND-3 $(a)$ и соответствующее флуоресцентное изображение капли $(b)$, полученное с использованием эпифлуоресцентного микроскопа и подсвечивающего лазера с длиной волны $532 \mathrm{~nm}$. Области интенсивного красного свечения соответствуют участкам на подложке, в которых реализуется наибольшая концентрация осажденных частиц FND-3. Диаметр кольца $0.8-0.9 \mathrm{~mm}$.

уширению линии разрешенного перехода и уменьшению ее пиковой интенсивности. Этот эффект усиливается с уменьшением размера частиц (вплоть до 5-10 nm) настолько, что в частицах размером 5-10 nm разрешенные переходы вообще не наблюдаются $[5,6]$. Не последнюю роль в уширении линии разрешенного перехода, повидимому, играет локальный разброс параметров спингамильтониана $D, E$ по ансамблю $\mathrm{NV}^{(-)}$центров в материале. Влияние механических напряжений, возникающих в кристаллитах, на числовые параметры спингамильтонианов для уровней ${ }^{3} \mathrm{~A}_{2}$ и ${ }^{3} \mathrm{E}$ детально обсуждалось в [15,27]. Анализ интегральных интенсивностей сигналов ЭПР $g_{1}=4.27$, полученных методом двойного интегрирования и отнесенных к массам исследуемых порошков, свидетельствует о концентрации флуоресцентных $\mathrm{NV}^{(-)}$центров в FND-1, FND-2 и FND-3 порядка $\sim 1.2, \sim 4$ и 5.5 ppm. Таким образом, метод ЭПР может быть использован для независимой оценки концентрации $\mathrm{NV}^{(-)}$центров во флуоресцентных алмазах размером менее 100-150 nm, где эти центры были специально (путем облучения пучками высокоэнергетических электронов) созданы.

\section{Люминесценция}

Спектры люминесценции FND-1, FND-2 и FND-3, получаемые при возбуждении лазерным излучением с длиной волны $532 \mathrm{~nm}$, демонстрируют характерные широкие полосы, связанные с эмиссией от $\mathrm{NV}^{(-)}$центров (рис. 6, кривые 1,2 и 3). Идентичность последних двух спектров проверялась их наложением и совмещением с использованием подгоночного масштабного коэффициента. На обоих спектрах (2 и 3) присутствуют идентичные особенности, отмеченные вертикальными пунктирными линиями (рис. 6). Материал с наиболее длительным временем экспозиции высокоэнергетическими электронами (FND-3), и наибольшей концентрацией

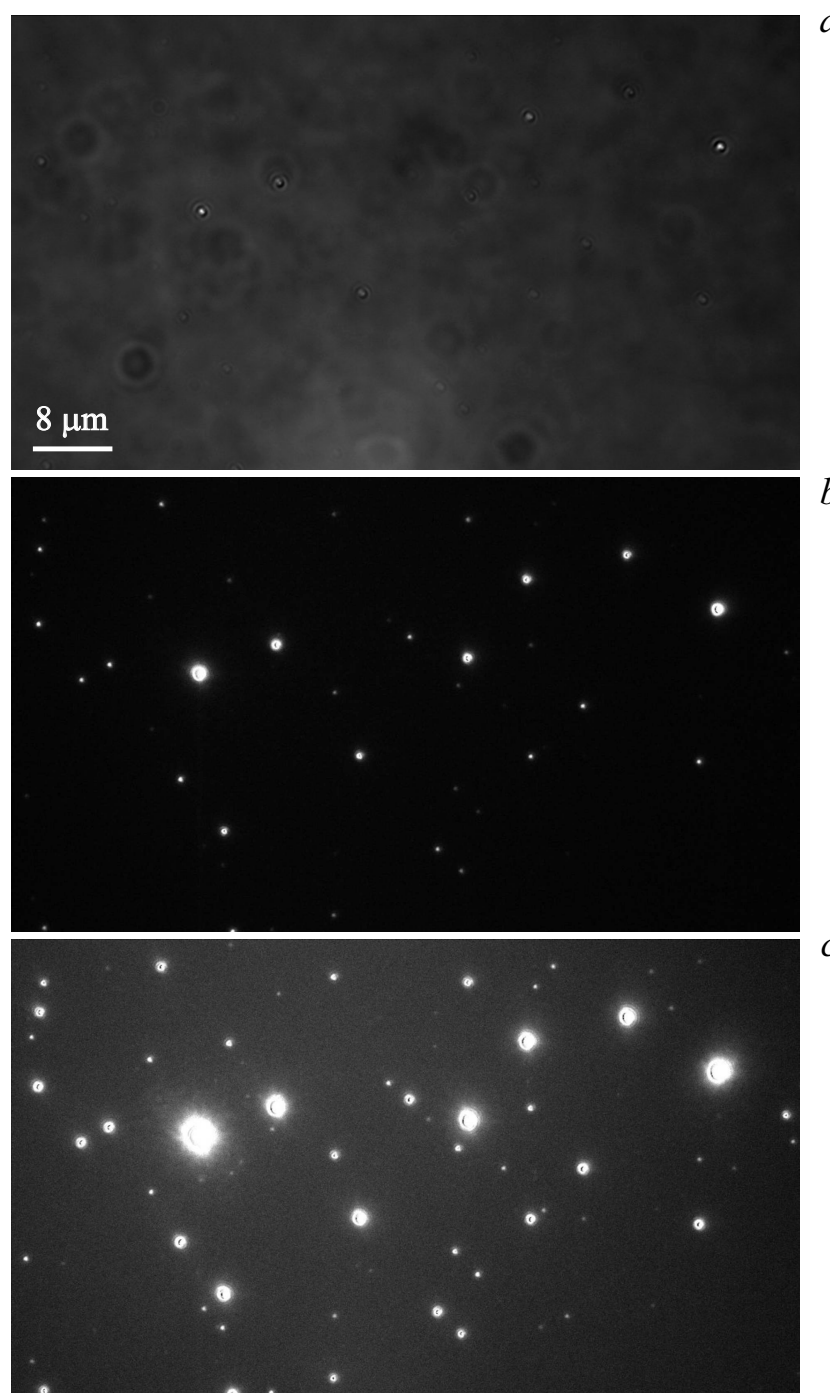

Рис. 8. Оптические и флуоресцентные изображения изолированных частиц FND-2 на стеклянной подложке, полученные с помощью разных методов оптической микроскопии: в режиме дифференциального интерференционного контраста при облучении белым светом $(a)$ и при облучении возбуждающим лазерным излучением на длине волны $561 \mathrm{~nm} \mathrm{c} \mathrm{временами}$ экспозиции снимка $60(b)$ и $600 \mathrm{~ms}(c)$.

$\mathrm{NV}^{(-)}$центров демонстрирует полосу люминесценции в интервале от 600 до $820 \mathrm{~nm}$, включающую узкую бесфононную линию при $637 \mathrm{~nm}$ и изломы в диапазоне 650-820 nm, связанные с фононными повторениями. Спектр такого вида практически полностью совпадает со спектром флуоресцентных синтетических кристаллов алмаза микронных размеров, полученных с использованием такой же техники облучения [28]. Интенсивность люминесценции FND-3 оказывается достаточной для практического использования. Флуоресцентное изображение высушенной капли суспензии наночастиц FND-3, полученное с помощью фильтра, пропускающего излучение в интервале 600-660 nm, показано на рис. 7. Оно свидетельствует о наличии более или менее однородно 
и ярко светящихся областей внутри кольца, размер которого задается диаметром капли. Интенсивность свечения этих областей пропорциональна концентрации осажденного в них материала при высыхании капли с суспензией. На рис. 8 представлены изображения осажденных на подложку изолированных частиц FND-2 и их агрегатов, полученные с помощью многофункционального микроскопа в режиме дифференциального интерференционного контраста при облучении белым светом (рис. 8, $a$ ), и в режиме флуоресценции при облучении $561 \mathrm{~nm}$ излучением и временами эскпозиции 60 и $600 \mathrm{~ms}$ (рис. 8, $b, c$ ). С увеличением времени экспозиции кадра до $600 \mathrm{~ms}$ становится видно больше частиц, имеющих меньшее число эмиссионных центров и не проявляющихся при экспозиции в $60 \mathrm{~ms}$. При этом на обоих флуоресцентных изображениях (рис. $8, b, c$ ) отлично проявляются малые частицы с низкой эмиссионной способностью, которые по фундаментальным соображениям не могут быть разрешены на дифференциальных интерференционноконтрастных изображениях, полученных в режиме на пропускание (рис. 8,a). Они имеют размеры меньшие $50 \mathrm{~nm}$ и соответственно меньшее количество эмиссионных $\mathrm{NV}^{(-)}$центров. В свою очередь наиболее яркие пятна на рис. $8, b, c$ обусловлены агрегатами из трех-четырех (и более) случайно аглютинированных $100 \mathrm{~nm}$ частиц. Превосходные флуоресцентные характеристики FND-3 обусловлены как высокой концентрацией $\mathrm{NV}^{(-)}$центров $(5.5 \mathrm{ppm})$, так и высоким качеством решетки алмаза в частицах-кристаллитах со средним размером $100 \mathrm{~nm}$. Средний поток фотонов, идущий от одной флуоресцентной частицы FND-3 в используемых (в данной работе) условиях оптического возбуждения, достаточно интенсивный и составляет около $\sim(9-10) \cdot 10^{6} \mathrm{kcs} / \mathrm{s}$. Если предположить, что один только $\mathrm{NV}^{(-)}$центр испускает квазистационарный поток фотонов величиной $\sim 20 \mathrm{kcs} / \mathrm{s}$, как это имеет место для малых частиц с одним центром внутри [1], то мы получим около $\sim 450-500 \mathrm{NV}^{(-)}$ центров в одной $\sim 100 \mathrm{~nm}$ частице FND-3. Оценки, сделанные из измерений интенсивности люминесценции, неплохо совпадают с оценками концентрации $\mathrm{NV}^{(-)}$, полученными из анализа интегральной интенсивности ЭПР сигнала $g_{1}=4.27$. Общее количество дефектов, индуцированных размолом, как в самой решетке, так и в механически поврежденной области вблизи поверхности (глубиной до 10-12 nm), является несущественным для гашения люминесценции $\mathrm{NV}^{(-)}$центров внутри таких $100 \mathrm{~nm}$ частиц, и может заранее контролироваться методом электронного парамагнитного резонанса. Изготовленные специально облученные и отожженные синтетические НРНТ кристаллы Ib алмаза $100 \mathrm{~nm}$ размера могут использоваться в микро-оптических устройствах, волоконных системах и сенсорах, работающих на принципах нанофотоники. По своим оптическим эмиссионным характеристикам они ненамного уступают объемным микрокристаллам алмаза с $\mathrm{NV}^{(-)}$центрами. Специальные исследования позволили установить, что изготовленные наночастицы могут использоваться при изучении биологических объектов: для оконтуривания внутриклеточных органелл [29] и трекинга движения клеток [30]. Излучение от частиц, введенных в подкожную область подопытных лабораторных животных, также легко регистрируется стандартными оптическими средствами.

\section{Заключение}

Синтетические наноалмазы с размером частиц $\sim 100 \mathrm{~nm}$, облученные в субмикронном морфологическом состоянии, а затем отожженные, демонстрируют высокую (до $5.5 \mathrm{ppm}$ ) концентрацию $\mathrm{NV}^{(-)}$центров одновременно с отсутствием заметного числа поверхностных дефектов (мультивакансий), наводимых исходным механическим размолом. Метод ЭПР с регистрацией слабых сигналов в половинном магнитном поле является эффективным не только для экспресс-оценки концентрации $\mathrm{NV}^{(-)}$центров в синтетических НРНT Ib флуоресцентных наноалмазах, но и для оценки качества кристаллической решетки в окрестности $\mathrm{NV}^{(-)}$центров по виду кривых насыщения амплитуд ЭПР сигналов, соответствующих запрещенным и разрешенным переходам в этих триплетных центрах. Кристаллическое качество решетки в $100 \mathrm{~nm}$ частицах флуоресцентного алмаза не сильно отличается от такового для объемного алмаза, и является достаточно приемлемым для получения яркой и сверхяркой люминесценции от таких частиц. Интегральная интенсивность сигнала ЭПР от $\mathrm{NV}^{(-)}$ центров, связанного с запрещенными переходами, коррелирует с интенсивностью люминесценции $\mathrm{NV}^{(-)}$центров в $100 \mathrm{~nm}$ частицах в спектральном диапазоне $600-800 \mathrm{~nm}$.

\section{Благодарности}

Авторы благодарят проф. Казуюки Такаи (Нosei University, Japan) за содействие в магнитных исследованиях. В.Ю.О. благодарит университет Ecole Normale Supérieure de Paris-Saclay (Gif-sur-Yvette, France) за поддержку в качестве приглашенного профессора и возможность проведения экспериментов по теме данной работы.

\section{Финансирование работы}

Эта работа была поддержана Российским научным фондом (соглашение 21-12-00264).

\section{Конфликт интересов}

Авторы декларируют отсутствие конфликта интересов в рамках исследования, изложенного в данной статье. 


\section{Список литературы}

[1] H.C. Chang, W.W.W. Hsiao, M.C. Su. Fluorescent nanodiamonds. (John Wiley \& Sons, Hoboken-Chichester-Oxford, 2019).

[2] S.J. Yu, M.W. Kang, H.C. Chang, K.M. Chen, Y.C. Yu. J. Am. Chem. Soc., 127 (50), 17604 (2005). DOI: 10.1021/ja0567081

[3] R. Schirhagl, K. Chang, M. Loretz, C.L. Degen. Annu. Rev. Phys. Chem., 65, 83 (2004). DOI: 10.1146/annurevphyschem-040513-103659

[4] O. Shenderova, G. McGuire. Biointerphases, 10, 030802 (2015). DOI: $10.1116 / 1.4927679$

[5] A.I. Shames, V.Y. Osipov, H.J. von Bardeleben, J.P. Boudou, F. Treussart, A.Y. Vul'. Appl. Phys. Lett., 104 (6), 063107 (2014). DOI: $10.1063 / 1.4865205$

[6] A.I. Shames, V.Y. Osipov, J.P. Boudou, A.M. Panich, H.J. von Bardeleben, F. Treussart, A.Y. Vul'. J. Phys. D: Appl. Phys., 48 (15), 155302 (2015). DOI: 10.1088/00223727/48/15/155302

[7] J.H.N. Loubser, J.A. van Wyk. Rep. Prog. Phys., 41, 1201 (1978). DOI: 10.1088/0034-4885/41/8/002

[8] A.M. Zaitsev Optical properties of diamond: $A$ data handbook. (Springer-Verlag, Berlin-Heidelberg-New York, 2001). DOI: $10.1007 / 978-3-662-04548-0$

[9] G. Davies, M.F. Hamer. Proc. R. Soc. Lond. A. Math. Phys. Sci., 348 (1653), 285 (1976). DOI: 10.1098/rspa.1976.0039

[10] V.Y. Osipov, F. Treussart, S.A. Zargaleh, K. Takai, F.M. Shakhov, B.T. Hogan, A. Baldycheva. Nanoscale Res. Lett., 14 (1), 1 (2019). DOI: 10.1186/s11671-019-3111-y

[11] G.K. Walters, T.L. Estle. J. Appl. Phys., 32, 1854 (1961). DOI: $10.1063 / 1.1728252$

[12] T. Rosskopf, A. Dussaux, K. Ohashi, M. Loretz, R. Schirhagl, H. Watanabe, S. Shikata, K.M. Itoh, C.L. Degen. Phys. Rev. Lett., 112, 147602 (2014). DOI: 10.1103/PhysRevLett.112.147602

[13] D.W. Boukhvalov, V.Y. Osipov, K. Takai. Phys. Chem. Chem. Phys., 23, 14592 (2021). DOI: 10.1039/D0CP05914E

[14] V.Y. Osipov, F.M. Shakhov, K.V. Bogdanov, K. Takai, T. Hayashi, F. Treussart, A. Baldycheva, B.T. Hogan, C. Jentgens. Nanoscale Res. Lett., 15 (1), 1 (2020). DOI: 10.1186/s11671-020-03433-7

[15] M.W. Doherty, V.V. Struzhkin, D.A. Simpson, L.P. McGuinness, Y. Meng, A. Stacey, T.J. Karle, R.J. Hemley, N.B. Manson, L.C.L. Hollenberg, S. Prawer. Phys. Rev. Lett., 112 (4), 047601 (2014). DOI: 10.1103/PhysRevLett.112.047601

[16] D.A. Broadway, B.C. Johnson, M.S.J. Barson, S.E. Lillie, N. Dontschuk, D.J. McCloskey, A. Tsai, T. Teraji, D.A. Simpson, A. Stacey, J.C. McCallum, J.E. Bradby, M.W. Doherty, L.C.L. Hollenberg, J.-P. Tetienne. Nano Lett., 19 (7), 4543 (2019). DOI: 10.1021/acs.nanolett.9b01402

[17] V.Y. Osipov, F.M. Shakhov, N.N. Efimov, V.V. Minin, S.V. Kidalov, A.Y. Vul'. Solid State Phys., 59 (6), 1146 (2017). DOI: $10.1134 / \mathrm{S} 1063783417060191$

[18] W.V. Smith, P.P. Sorokin, I.L. Gelles, G.J. Lasher. Phys. Rev., 115, 1546 (1959). DOI: 10.1103/PhysRev.115.1546

[19] B.R. Smith, D.W. Inglis, B. Sandnes, J.R. Rabeau, A.V. Zvyagin, D. Gruber, C.J. Noble, R. Vogel, E. Osawa, T. Plakhotnik. Small, 5, 1649 (2009). DOI: $10.1002 / \mathrm{smll} .200801802$

[20] V.Y. Osipov, A.I. Shames, T. Enoki, K. Takai, M.V. Baidakova, A.Y. Vul'. Diam. Relat. Mat., 16(12), 2035 (2007). DOI: 10.1016/j.diamond.2007.06.003
[21] S. Sotoma, D. Terada, T.F. Segawa, R. Igarashi, Y. Harada, M. Shirakawa. Sci. Rep., 8 (1), 1 (2018). DOI: $10.1038 / \mathrm{s} 41598-018-23635-5$

[22] Á Gali. Nanophotonics, 8 (11), 1907 (2019). DOI: $10.1515 /$ nanoph-2019-0154

[23] S.D. Subedi, V.V. Fedorov, J. Peppers, D.V. Martyshkin, S.B. Mirov, L. Shao, M. Loncar. Opt. Mater. Express, 9(5), 2076 (2019). DOI: 10.1364/OME.9.002076

[24] M.W. Doherty, N.B. Manson, P. Delaney, F. Jelezko, J. Wrachtrup, L.C.L Hollenberg. Phys. Rep., 528 (1), 1 (2013). DOI: 10.1016/j.physrep.2013.02.001

[25] A.I. Shames, V.Y. Osipov, H.J. von Bardeleben, A.Y. Vul'. J. Phys.: Condens. Matter., 24 (22), 225302 (2012). DOI: $10.1088 / 0953-8984 / 24 / 22 / 225302$

[26] S. Felton, A.M. Edmonds, M.E. Newton, P.M. Martineau, D. Fisher, D.J. Twitchen, J.M. Baker. Phys. Rev. B., 79(7), 075203 (2009). DOI: 10.1103/PhysRevB.79.075203

[27] M.W. Doherty, N.B. Manson, P. Delaney, L.C.L. Hollenberg. New J. Phys., 13 (2), 025019 (2011). DOI: 10.1088/13672630/13/2/025019

[28] A.I. Shames, V.Y. Osipov, K.V. Bogdanov, A.V. Baranov, M.V. Zhukovskaya, A. Dalis, S.S. Vagarali, A. Rampersaud. J. Phys. Chem. C., 121 (9), 5232 (2017). DOI: $10.1021 /$ acs.jpcc.6b12827

[29] I. Rehor, J. Slegerova, J. Kucka, V. Proks, V. Petrakova, M.P. Adam, F. Treussart, S. Turner, S. Bals, P. Sacha, M. Ledvina, A.M. Wen, N.F. Steinmetz, P. Cigler. Small, 10 (6), 1106 (2014). DOI: $10.1002 / \mathrm{smll} .201302336$

[30] S. Haziza, N. Mohan, Y. Loe-Mie, A.M. Lepagnol-Bestel, S. Massou, M.P. Adam, X.L. Le, J. Viard, C. Plancon, R. Daudin, P. Koebel, E. Dorard, C. Rose, F.-J. Hsieh, C.C. Wu, B. Potier, Y. Herault, C. Sala, A. Corvin, B. Allinquant, H.-C. Chang, F. Treussart, M. Simonneau. Nat. Nanotechnol., 12 (4), 322 (2017). DOI: 10.1038/nnano.2016.260 